\title{
Observation of Kerr nonlinearity in microcavities at room temperature
}

\author{
Hossein Rokhsari and Kerry J. Vahala \\ Thomas J. Watson Laboratories of Applied Physics, California Institute of Technology, Pasadena, California 91125
}

Received August 19, 2004

\begin{abstract}
We have devised and experimentally verified a method for observation of the optical Kerr effect in microcavities at room temperature. The technique discriminates against the much larger and typically dominant thermal component of nonlinearity by using its relatively slow frequency response. Measurement of the Kerr coefficient or equivalently of the third-order nonlinear susceptibility of the cavity material is demonstrated for a silica microcavity. With this approach, useful information about the characteristic thermal response time in microresonators can also be acquired. (C) 2005 Optical Society of America

OCIS codes: 190.3270, 190.4360, 190.1900, 230.5750.
\end{abstract}

Ultrahigh-quality factor (high-Q) whispering gallery mode (WGM) microcavities (e.g., microspheres and microtoroids) are ideally suited for observing nonlinear optical effects with extremely low (submicrowatt) threshold powers. ${ }^{1-5}$ The combination of high circulating power made possible by the high quality factor and strong confinement of this power within mode volumes of the order of hundreds of cubic micrometers leads to intercavity circulating intensities in excess of gigawatts per square centimeter with only $1 \mathrm{~mW}$ of input power (assuming $Q$ values of approximately 100 million in microtoroid resonators with principal diameters of $\sim 50 \mu \mathrm{m}$ ). This level of intensity is sufficient to unveil nonlinear phenomena even in materials such as silica that are weakly nonlinear. The optical Kerr effect and a variety of its applications have been the subject of numerous research activities around the world. ${ }^{6}$ The observation of this phenomenon in microcavities, however, is problematic, as it is accompanied by relatively larger thermal nonlinear effects. ${ }^{4,5,7}$ The refractive index of silica, which is also a function of temperature, varies as the circulating power is partially absorbed by the medium, and the cavity temperature subsequently increases. This larger effect (thermo-optic effect) masks the Kerr-driven variations in the refractive index. Treussart et al. ${ }^{5}$ found evidence for Kerr bistability in microresonators by immersing microspheres in a superfluid helium bath at $2 \mathrm{~K}$. This cryogenic setup greatly reduces the sensitivity of WGM resonances to temperature and allows the Kerr effect to be dominant and hence observable.

In this Letter we propose and experimentally verify a method for observing the optical Kerr effect in microcavities at room temperature. This technique also allows us to estimate the $\chi^{(3)}$ or equivalently the $n_{2}$ coefficient in silica. The method relies on the relatively long response times of thermal effects compared with that of the almost instantaneous Kerr effect. To manipulate this distinction in favor of the Kerr effect we use two beams (pump and probe) individually resonant with two WGM resonances of an optical microcavity. Modulation of the pump power results in modulation of the cavity's refractive index and consequently in modulation of the resonant frequency of the WGM with which the probe beam is in resonance. The probe beam's power transmission beyond the resonator waveguide junction therefore acquires a strong Fourier component at the modulation frequency of the pump beam. This component can be measured with a lock-in amplifier. When the pump's modulation frequency becomes sufficiently faster than the thermal effects, the signal detected by the lock-in amplifier is due purely to the Kerr effect.

The modulated probe power (with frequency $\Omega$ ) is given by the following equation:

$$
\frac{\Delta P_{\text {probe }}^{\Omega}}{P_{\text {probe }}}=P_{\text {pump }}^{\Omega} Q_{\text {probe }}^{\text {total }} Q_{\text {pump }}^{\text {eff }} \frac{2 n_{2} \lambda_{\text {pump }}}{\pi n^{2} V_{\text {eff }}} C(x),
$$

where $P_{\text {probe }}$ is the probe power coupled into the resonator and $P_{\text {pump }}^{\Omega}$ is the fraction of pump power coupled into the cavity and carrying modulation frequency $\Omega$. The modulated probe power $\left(\Delta P_{\text {probe }}^{\Omega}\right)$ depends on the refractive-index change in the cavity caused by the modulated pump power $\left(P_{\text {pump }}^{\Omega}\right)$, which is proportional to the $n_{2}$ coefficient, the quality factor of the cavity at pump wavelength $\left(Q_{\text {pump }}^{\text {eff }}\right)$, and the cross section of the optical mode. The changes in refractive index of the cavity result in variation of the probe power's transmission, which depends greatly on the slope of the transmission profile versus the linewidth or, in other words, the quality factor of the WGM coupled to the probe signal. The total quality factor of the optical mode at the probe frequency ( $\left.\nu_{\text {probe }}\right)$ is given by $Q_{\text {probe }}^{\text {total }}$, which includes both intrinsic losses of the cavity and loading by the optical waveguide and can be obtained from linewidth measurements in the desired coupling regime. Transfer of modulation from pump to probe beam also depends on detuning of the cavity's resonant frequency from that of the probe beam, which, measured in units of cavity linewidth, is denoted $x$. The functional form of this dependence is given by $C(x)=2 x / 1+x^{2}$, a number between 1 and 0 depending on the value of $x$. The effective quality factor $\left(Q_{\text {pump }}^{\text {eff }}\right.$, defined below $)$ of the resonator at the pump wavelength $\left(\lambda_{\text {pump }}\right)$ determines the enhancement 
of the pump power in the cavity ${ }^{8}$ :

$$
\frac{P_{\text {pump }}^{\text {cavity }}}{P_{\text {pump }}}=\frac{\lambda_{\text {pump }} Q_{\text {pump }}^{\text {eff }}}{\pi^{2} n R}
$$

where $R$ and $n$ denote the radius and the refractive index, respectively, of the cavity. Equation (3) below shows how $Q_{\text {pump }}^{\text {eff }}$ can be obtained from coupling parameter $K$, defined as $K=Q_{0} / Q_{\text {ext }}$, where $Q_{0}$ is the intrinsic quality factor of the optical mode and $Q_{\text {ext }}$ is the quality factor associated with the coupling to the optical waveguide:

$$
Q_{\mathrm{pump}}^{\mathrm{eff}}=Q_{0} \frac{K}{(1+K)^{2}} .
$$

Finally, note the role of small mode volumes $\left(V_{\text {eff }}\right)$ in Eq. (1) in reducing the required optical power for observation of nonlinear effects.

The experimental setup that we used to observe and measure the Kerr nonlinearity of silica microcavities is shown in Fig. 1. Two tunable external-cavity diode lasers were used to generate pump and probe beams in the 1550- and 1480-nm bands, respectively. The pump and probe laser frequencies are simultaneously tuned to two optical WGM resonances of a toroidal microcavity ${ }^{9}$ and launched into a tapered optical fiber by a WDM coupler. Tapered optical fibers with waist diameters of $1-3 \mu \mathrm{m}$ were fabricated by the traditional technique of simultaneous flame heating and pulling of standard single-mode (SMF-28) fibers. ${ }^{10}$ Efficient and ideal coupling to the optical modes of microtoroid cavities is possible with these waveguides. ${ }^{11}$ A top-view optical micrograph of a microtoroid evanescently side coupled to a tapered optical fiber is shown in Fig. 1. The output of the 1550-nm laser is modulated with a Mach-Zehnder modulator (bandwidth, $20 \mathrm{GHz}$ ) driven by a rf function generator (Func. Gen.) that generates sinusoidal waves with frequencies as high as $80 \mathrm{MHz}$. Because of residual transmitted modulated pump power in the probe channel, the detected signal could potentially mask the relatively small modulation in the probe power, and therefore two cascaded wavelength-selective couplers are used to ensure high extinction (above $38 \mathrm{~dB}$ ) of the pump power in the probe channel. The pump and probe laser frequencies are adjusted while the detected pump and probe transmissions are monitored by an oscilloscope to ensure that both frequencies are tuned to the desired proximity of the resonant frequencies of the cavity. The probe laser was slightly detuned from resonance to maintain $50 \%$ transmission through the junction [i.e., $C(x)=0.8$ ]. The microtoroid is coupled almost critically to both pump and probe signals in these experiments. A high-precision piezoelectric transducer stage (10-nm steps) was used to position the microtoroid relative to the tapered fiber and thereby to control the coupling to the resonator. With the laser frequencies tuned to proximity of the desired WGM resonances and with the pump power modulated, the probe power is detected and the photocurrent is processed by the lock-in amplifier with the output of the function generator as its reference signal. The amplifier was operated in the $(r, \theta)$ setting, where $r$ (in volts) and $\theta$ (in degrees) are the amplitude and the relative phase of the detected signal at the reference frequency $(\Omega)$.

The output of the lock-in amplifier ( $r$, in microvolts) is plotted in Fig. 2 versus the modulation frequency. This plot is normalized to the frequency response of the entire system with the microtoroid decoupled from the fiber taper. Therefore the plot shown in Fig. 2 is the pure response that results from cross modulation of the probe beam by the pump beam. Thermal effects can follow the modulation of the pump power up to $\sim \Omega=10 \mathrm{kHz}$; hence a flat response is observed

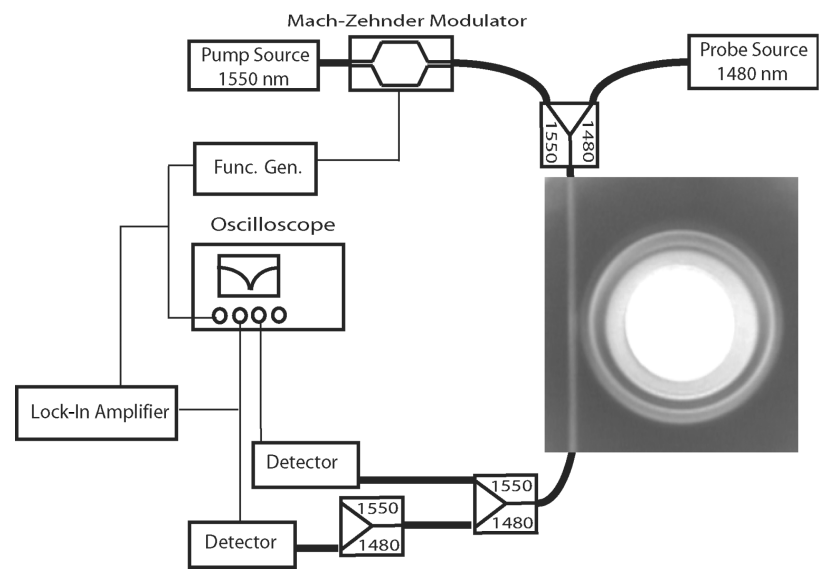

Fig. 1. Experimental setup used for characterizing the Kerr nonlinearity of microcavities. The picture is a topview optical micrograph of a toroid microresonator that has been evanescently side coupled to a tapered optical fiber. Laser sources at 1550- and 1480-nm bands are used for pump and probe beams, respectively.

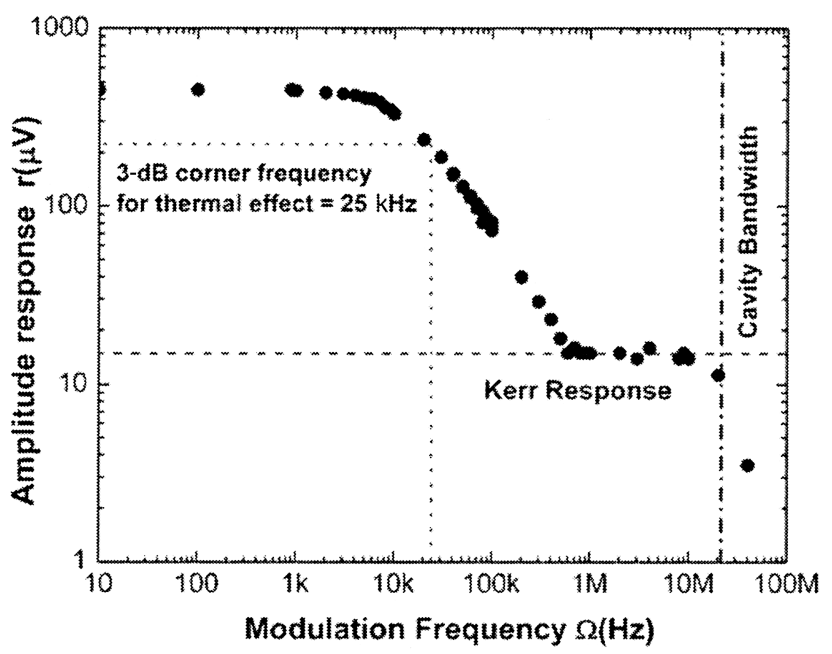

Fig. 2. Measured amplitude modulation of the probe beam as a function of the modulation frequency of the pump power. The dotted lines show a 3-dB corner frequency of $\sim 25 \mathrm{kHz}$ where the modulation of the pump power becomes comparable to or faster than the thermal response time of the resonator. The second roll-off is due to limited bandwidth of the cavity, which does not allow the pump power in the resonator to build up instantaneously. The flat response in the middle shows the fast Kerr effect. 
below this frequency. At higher frequencies, however, a decline in the amplitude response is observed, with a 3-dB corner frequency of $\sim 25 \mathrm{kHz}$, suggesting a thermal response time of the order of $6 \mu \mathrm{s}$. As was mentioned in Ref. 4, the fastest thermal response time is associated with the conduction of heat generated in the optical mode volume to the cavity bulk material and can be put into the following compact form:

$$
\tau_{\text {thermal }}=\frac{(\delta R)^{2}}{D}=\frac{1}{D}\left(\frac{2 R \lambda^{2}}{\pi^{2} n^{2}}\right)^{2 / 3}
$$

where $\delta R$ is the effective thickness of the optical mode localized near the surface of the cavity and $D$ is the temperature conductivity of glass $\left(8 \times 10^{-3} \mathrm{~cm}^{2} / \mathrm{s}\right)$. For the microtoroid under study here with a major radius of $36 \mu \mathrm{m}$ and a minor diameter or thickness of $\sim 8 \mu \mathrm{m}$, the toroid geometry becomes similar to that of a sphere with respect to thermal effects within the modal volume. Applying Eq. (4) to this sample predicts a thermal time constant of $5 \mu \mathrm{s}$, in close agreement with the value derived from Fig. 2 .

Returning to Fig. 2, we can see that the decline in the amplitude response continues until it reaches a second plateau from $\sim 600 \mathrm{kHz}$ to $10 \mathrm{MHz}$ that is far beyond the modulation speeds that thermal effects can follow. To ensure that this flat response was not due to the noise level that arose from residual pump power in the 1480-nm channel or from rf cross talk, we switched off the probe power during data acquisition in the flat portion of the spectrum. This resulted in a decrease in the lock-in response by a factor of 40 , thereby confirming that the measured response is associated with modulation of the probe wave. Also, when the probe beam was detuned far away from resonance $[C(x) \approx 0]$, the signal dropped to the noise level, demonstrating that cross modulation of pump and probe occurs only in the cavity. Furthermore, by calibrating the lock-in amplifier we could infer the modulated probe power (i.e., $\Delta P_{\text {probe }}^{\Omega}$ ). Plugging this inferred power into Eq. (1), we obtain an $n_{2}$ value of $(6 \pm 4) \times 10^{-16} \mathrm{~cm}^{2} / \mathrm{W}$, which agrees well with measured $n_{2}$ values for fused silica $\left(n_{2}=3 \times 10^{-16} \mathrm{~cm}^{2} / \mathrm{W}\right)$. We therefore attribute the flattened response region in Fig. 2 to Kerr nonlinearity.

The optical modes coupled to the pump and probe beams had total quality factors of $10^{7}$ and $2 \times 10^{6}$, respectively. The power levels coupled into the resonator to generate the curve in Fig. 2 were $\sim 15$ and $\sim 10 \mu \mathrm{W}$ for pump and probe beams, respectively. Equation (1) shows that the signal level at frequency $\Omega$ can be preserved for lower- $Q$ optical modes at a cost of higher optical input powers. We confirmed this fact by repeating the same measurement for lower-quality factor WGM resonances both within the same cavity and within other microtoroids. Not only has the flat
Kerr response been observed and the inferred $n_{2}$ coefficient obtained in lower $Q$ samples but also the probe-to-pump power ratio has been reduced to less than $5 \%$, which completely rules out the possibility of feedback from the probe to the pump.

The flat Kerr response in the plot ultimately falls off because of the limited bandwidth of the optical mode. The optical field in the cavity is built up in a finite period that is roughly equal to the inverse of the optical mode linewidth. At modulation frequencies higher than the cavity linewidth the modulation of the pump power cannot fully build up within the cavity and be transferred to the probe beam. The WGM excited by the pump in the sample shown in Fig. 2 had a loaded $Q$ of $\sim 10^{7}$, which translates to a linewidth of $\sim 20 \mathrm{MHz}$ at $1550 \mathrm{~nm}$. By using lower- $Q$ optical modes, on the other hand, we could extend the Kerr plateau to higher frequencies.

In conclusion, in the present research we have demonstrated a powerful approach to observing Kerr nonlinearities in microcavities with extremely low optical powers. The simple experimental setup and room-temperature operation of these measurements are attractive for the study of nonlinear phenomena in microcavity structures. The method discussed is capable of measuring the nonlinear susceptibility of the cavity material and can be used to characterize the $n_{2}$ coefficients of various materials packaged in a microcavity type structure.

This research was supported by the Defense Advanced Research Projects Agency, the Caltech Lee Center, and the National Science Foundation. H. Rokhsari's e-mail address is rokhsari@caltech.edu.

\section{References}

1. R. K. Chang and A. J. Campillo, eds., Optical Processes in Microcavities (World Scientific, Singapore, 1996).

2. S. M. Spillane, T. J. Kippenberg, and K. J. Vahala, Nature 415, 621 (2002).

3. V. S. Ilchenko, A. A. Savchenkov, A. B. Matsko, and L. Maleki, Phys. Rev. Lett. 92, 043903 (2004).

4. V. S. Ilchenko and M. L. Gorodetsky, Laser Phys. 2, 1004 (1992).

5. F. Treussart, V. S. Ilchenko, J.-F. Roch, J. Hare, V. Lefevre-Seguin, J. M. Raimond, and S. Harche, Eur. Phys. J. D 1, 235 (1998).

6. R. W. Boyd, Nonlinear Optics (Academic, Boston, Mass., 1992).

7. V. B. Braginsky, M. L. Gorodetsky, and V. S. Ilchenko, Phys. Lett. A 137, 393 (1989).

8. H. Haus, Waves and Fields in Optoelectronics (Prentice-Hall, Englewood Cliffs, N.J., 1984).

9. D. K. Armani, T. J. Kippenberg, S. M. Spillane, and K. J. Vahala, Nature 421, 925 (2003).

10. M. Cai, O. J. Painter, and K. J. Vahala, Phys. Rev. Lett. 85, $74(2000)$.

11. S. M. Spillane, T. J. Kippenberg, O. J. Painter, and K. J. Vahala, Phys. Rev. Lett. 91, 043902 (2003). 